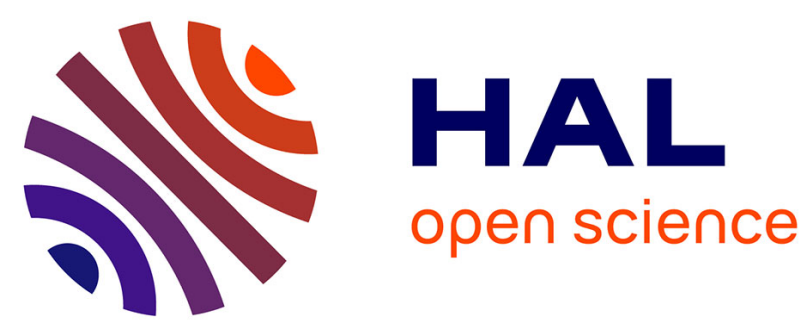

\title{
Influence of the operating frequency on DC-DC converters for HVDC grids
}

\author{
J. D Páez, J. Maneiro, S. Bacha, D. Frey, Piotr Dworakowski
}

\section{To cite this version:}

J. D Páez, J. Maneiro, S. Bacha, D. Frey, Piotr Dworakowski. Influence of the operating frequency on DC-DC converters for HVDC grids. 2019 21st European Conference on Power Electronics and Applications (EPE '19 ECCE Europe), Sep 2019, Genova, Italy. 10.23919/EPE.2019.8915433 . hal03303124

\section{HAL Id: hal-03303124 \\ https://hal.science/hal-03303124}

Submitted on 28 Jul 2021

HAL is a multi-disciplinary open access archive for the deposit and dissemination of scientific research documents, whether they are published or not. The documents may come from teaching and research institutions in France or abroad, or from public or private research centers.
L'archive ouverte pluridisciplinaire HAL, est destinée au dépôt et à la diffusion de documents scientifiques de niveau recherche, publiés ou non, émanant des établissements d'enseignement et de recherche français ou étrangers, des laboratoires publics ou privés. 


\title{
Influence of the operating frequency on DC-DC converters for HVDC grids
}

\author{
J. D. Páez ${ }^{1,2}$, J. Maneiro ${ }^{1}$, S. Bacha ${ }^{1,2}$, D. Frey ${ }^{1,2}$, P. Dworakowski ${ }^{1}$ \\ ${ }^{1}$ SuperGrid Institute \\ ${ }^{2} \mathrm{G} 2 \mathrm{ELab}$ \\ 23 Rue de Cyprian \\ 69100 Villeurbanne, France \\ juan.paez@supergrid-institute.com \\ https://www.supergrid-institute.com/ \\ Univ. Grenoble Alpes, CNRS, Grenoble INP* \\ 21 Avenue des Martyrs \\ 38000 Grenoble, France \\ http://www.g2elab.grenoble-inp.fr/ \\ *Institute of Engineering Univ. Grenoble Alpes
}

\section{Acknowledgements}

This work was supported by a grant overseen by the French National Research Agency (ANR) as part of the "Investissements d'Avenir" Program ANE-ITE-002-01.

\section{Keywords}

«High Voltage Power Converters», «HVDC», «DC-DC power converters»

\begin{abstract}
While several DC-DC converters for HVDC have been proposed in literature, comparison studies are needed to identify the best circuit for a particular case of application. This paper proposes an analytical methodology that allows to assess rapidly the comparison of DC-DC converters. It was applied to evaluate two modular DC-DC structures, one isolated circuit and one non isolated circuit, focusing in the variation of the operating frequency for different $\mathrm{DC}$ voltage transformation ratios. The results show that the non-isolated structure presents better indicators compared to the isolated circuit for all the considered cases.
\end{abstract}

\section{Introduction}

HVDC grids have been identified as a potential upgrade of the existing AC system. They offer flexibility and can be a solution for the integration of large-scale renewable energy sources. Given the differences in the existing and planned HVDC corridors, DC-DC converters will be required for the development of such grids [1]. These devices can interconnect grids with different voltage levels, technology (LCC/VSC) and architectures (monopole/bipole). In addition to the voltage adaptation, they offer other features as power flow control, DC voltage regulation, and could serve as firewalls against faults [2].

Recent research on DC-DC converters for HVDC tends to adapt the Modular Multilevel Converter (MMC) to DC-DC conversion [3]. The majority of circuits use MMC submodule (SM) arrangements. However, from the different proposals there is not a final choice of which circuit presents the best features for a given application, although some comparative studies have been reported [4]-[6].

In these studies the circuits are compared in terms of capital investment and losses. Some studies have been oriented to specific application cases [4], [6] while some others have been done using a more general approach [5]. For a final choice of the best converter candidate for a given application the first approach is more suitable, optimizing each structure for the application requirements. Nevertheless, these studies require a previous pre-selection of promising topologies. This pre-selection can be done using the second approach to identify the potential of each converter.

In [5], a comparison of HVDC DC-DC converters using this general approach has been done varying the DC voltage transformation ratio. However the study omitted the effect of the operation frequency. 
Indeed, since the AC quantities in a DC-DC structure are internal, the converter operation frequency can be freely chosen. Given the differences on the operation principle of each topology, the effects of changing the frequency can make that the conclusions of which topology presents less capital cost and losses can vary. In this paper, a general methodology to compare modular multilevel DC-DC converters is proposed. The methodology is then applied to compare two modular DC-DC converters focusing on the effects of varying the operation frequency for different DC voltage transformation ratios.

\section{Modular DC-DC converter topologies}

The most straightforward way to achieve DC-DC conversion is to implement a DC-AC-DC conversion scheme using an existing AC-DC conversion technology. The Front-to-Front MMC (F2F-MMC) [7] uses this principle: two MMCs are interconnected on the AC side through an $\mathrm{AC}$ transformer as shown in Fig. 1a. For its operation each MMC generates an AC voltage on the AC terminals and the power transfer is achieved controlling the phase shift. This topology provides galvanic isolation thanks to the presence of the AC transformer.

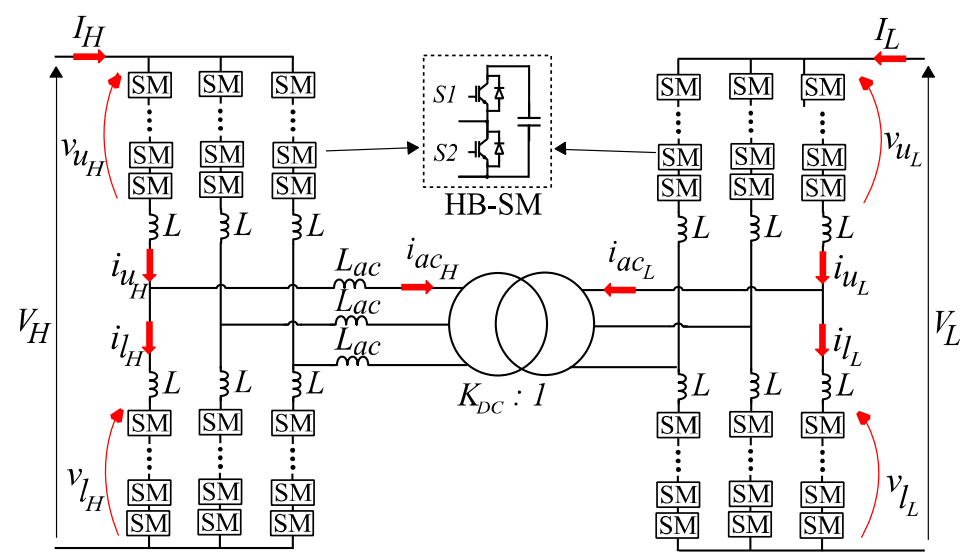

a. Front-to-Front MMC (F2F-MMC)

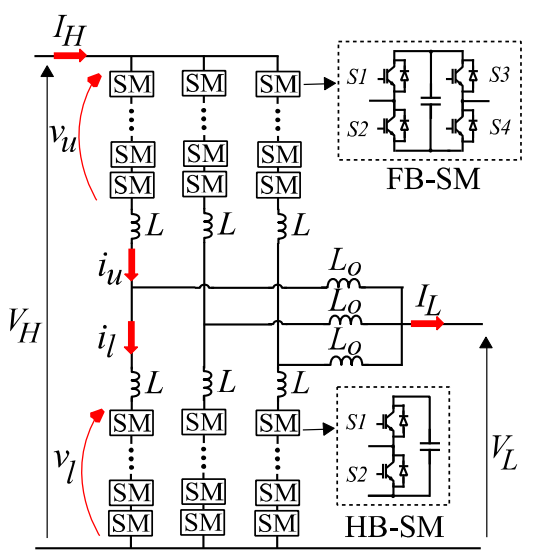

b. DC-MMC

Fig. 1 - Two modular three-phase DC-DC converters.

As alternative to the F2F-MMC, the DC-MMC [8], [9] is implemented with only one MMC interconnecting the three output AC phases through an AC filter as presented in Fig. 1b. In the example, the filter is constituted of three single phase inductors, but different filters can be used [10]. The converter operation is based on the generation of DC and AC currents inside the converter. The DC currents are responsible for the DC power transfer while the AC current circulates internally to balance the arm energies. This topology does not provide galvanic isolation but can implement fault blocking capability like the F2F-MMC if full-bridge (FB) SMs are used on the upper arms. If such feature is not required the topology can work with half-bridge (HB) SMs. In the present study it will be assumed that the topology can block faults as the F2F-MMC.

Table I: Parameters for comparison study

\begin{tabular}{|l|l|}
\hline DC Power $P_{D C}$ & $700 \mathrm{MW}$ \\
\hline High voltage $V_{H}$ & $640 \mathrm{kV}$ \\
\hline Low voltage $V_{L}$ & Determined by $K_{D C}$ \\
\hline Transformation ratio $K_{D C}=V_{H} / V_{L}$ & $1.3,2,2.7$ \\
\hline AC frequency $f$ & Varied from $50 \mathrm{~Hz}$ to $350 \mathrm{~Hz}$ in steps of $50 \mathrm{~Hz}$ \\
\hline Arm inductances $L$ & $15 \mathrm{mH}$ \\
\hline AC inductance $L_{a c}(\mathrm{~F} 2 \mathrm{~F}-\mathrm{MMC})$ & $20 \mathrm{mH}$ \\
\hline Output inductance $L_{o}$ (DC-MMC) & $150 \mathrm{mH}$ \\
\hline SM Capacitors $C_{S M}$ & Sized to have $10 \%$ voltage ripple \\
\hline SM mean voltage $\overline{V_{S M}}$ & $1.6 \mathrm{kV}$ \\
\hline
\end{tabular}




\section{Comparison methodology}

The proposed converter comparison is based on three indicators: utilization factor of semiconductors, energy storage requirements, and losses. It is proposed to calculate these indicators analyzing the operation of each circuit in steady state. From this analysis the expressions of the DC and AC quantities (voltage and currents) per converter arm are obtained. The analysis assumes only an AC component at the fundamental frequency. With these expressions each indicator can be calculated and applied to compare both circuits fixing the DC power and the High DC voltage as presented in Table I. The DC voltage transformation ratio and operation frequency are varied.

\section{Utilization Factor}

The utilization factor is defined by the ratio between transmitted power and the total VA rating of the semiconductor switches:

$$
U_{S W}=\frac{P_{D C}}{\sum V_{S W_{p k}} I_{S W_{R M S}}}
$$

This factor reflects the investment in semiconductors, since for higher VA rating devices with higher ratings are needed. Then the topologies with a higher $U_{S W}$ are preferable.

To calculate the stress on each semiconductor the peak voltage and the RMS current per switch are needed. The switch peak voltage is equal to the peak voltage on the SM capacitor. Since the capacitors in this study are sized to have a ripple of $10 \%$, it means that the peak voltage value is:

$$
V_{S W_{p k}}=V_{C_{p k}}=\overline{V_{S M}}+\Delta V=1.1 \overline{V_{S M}}
$$

The switch RMS currents are difficult to estimate since the duty cycle in each SM is variable, a method to address this issue is explained later on the converter losses section. From that procedure the RMS current on all the switches in one SM is estimated, then the maximum value between them is used as rating for all the switches in the same SM. This value is used for the calculation of Eq. (1).

\section{Energy Requirements}

The energy requirements of modular DC-DC converters are mainly due to the installed capacitance in the converter SMs, and can be resumed in an energy factor as follows:

$$
E=\frac{W_{\text {installed }}}{P_{D C}}=\frac{\sum \frac{1}{2} C_{S M}{\overline{V_{S M}}}^{2}}{P_{D C}}
$$

This factor reflects the investment in SM capacitors and in some way the volume of the converter since the volume of one SM is mainly due to the capacitor size. Then the topologies with a lower $E$ are preferable. In the present study the converter capacitors will be sized to the minimum value to have $10 \%$ of voltage ripple at nominal power.

The SM capacitor sizing depends on the maximum energy variation experienced by a SM and it can be calculated generalizing the procedure proposed in [11] for the MMC, obtaining:

$$
C_{S M}=\frac{\frac{V_{D C_{a r m}} I_{D C_{a r m}}}{2 \pi f}\left(1-\left(\frac{k \cos \phi}{2}\right)^{2}\right)^{\frac{3}{2}} \frac{4}{k \cos \phi}}{2 \varepsilon N_{S M}{\overline{V_{S M}}}^{2}}
$$

Where $V_{D C_{\text {arm }}}$ and $I_{D C_{\text {arm }}}$ represent the DC quantities on each arm, $k$ is a factor representing the ratio between the arm AC peak voltage and the arm DC voltage, i.e. the modulation index, $\phi$ is the phase angle between arm current and voltage, $N_{S M}$ is the number of submodules on the arm and $f$ represents the operation frequency.

\section{Converter Losses}

While the $U_{S W}$ and $E$ indicators allow to compare the converter capital cost and size, the converter losses are selected as the key indicator to evaluate their operation costs related to the efficiency. It is proposed to evaluate only the losses on semiconductors. Then two kind of losses are evaluated: conduction and 
switching losses. The losses on each switch are calculated and then added to obtain a loss factor normalizing by the transmitted DC power:

$$
P_{S W}=\frac{\sum P_{\text {switching }_{S w}}}{P_{D C}} \quad P_{\text {cond }}=\frac{\sum P_{\text {conduction }_{S w}}}{P_{D C}}
$$

These factors need the losses estimation on each switch on the SMs. For conduction losses on a semiconductor switch (IGBT or diode), they are approximated by:

$$
P_{\text {cond }_{S W}}=V_{0} I_{\text {avg }}+R_{O N} I_{R M S}^{2}
$$

Where $V_{0}$ represent the saturation voltage if the switch is an IGBT or the threshold voltage if it is a diode, and $R_{O N}$ represents the device equivalent resistance in the ON state. Both values can be obtained from the device datasheet, in this study an Infineon pack of 3.3kV-1500A (FZ1500R33HL3) with a junction temperature of $125^{\circ} \mathrm{C}$ is considered. The currents $I_{\text {avg }}$ and $I_{R M S}$ are the average and RMS values of the current flowing through the device in the ON state.

Since in a modular structure based on SMs the duty cycle is variable and different on each SM, it is difficult to determine the average and RMS currents on each switch. However, an estimation can be done following the analysis proposed in [12], [13] where averaged currents are used. In [14] the same method is used but applied directly on the conduction losses. According to [12] during a small interval $\alpha(t) T_{s}$ where $T_{S}$ is the switching period of the SM and $\alpha(t)$ the duty cycle, the current on the switch is constant then:

$$
\begin{gathered}
\left\langle I_{S W_{\mathrm{AVG}}}(t)\right\rangle=\frac{1}{T_{s w}} \int_{t_{o}}^{t_{o}+\alpha(t) T} I_{S w}(t) d \tau=I_{S w}(t) \alpha(t) \\
\left\langle I_{S W_{R M S}}(t)\right\rangle=\sqrt{\frac{1}{T_{S w}} \int_{t_{o}}^{t_{o}+\alpha(t) T}\left(I_{s w}(t)\right)^{2} d \tau}=I_{S W}(t) \sqrt{\alpha(t)}
\end{gathered}
$$

From these values according to [12], [13], the average and RMS current on a switch are:

$$
\begin{gathered}
I_{S W_{\mathrm{AVG}}}=\frac{1}{T} \int_{t_{1}}^{t_{2}}\left\langle I_{S W_{\mathrm{AVG}}}(t)\right\rangle d t=\frac{1}{T} \int_{t_{1}}^{t_{2}} i_{\text {arm }}(t) \alpha(t) d t \\
I_{S W_{R M S}}=\sqrt{\frac{1}{T} \int_{t_{1}}^{t_{2}}\left\langle I_{S w_{R M S}}(t)\right\rangle^{2} d t}=\sqrt{\frac{1}{T} \int_{t_{1}}^{t_{2}}\left(i_{\text {arm }}(t)\right)^{2} \alpha(t) d t}
\end{gathered}
$$

According to the current polarity and the duty cycle, which is proportional to the voltage being generated by the arms [12], it is possible to identify which semiconductor is conducting at any time according to Table II. Therefore the integration on Eqs (9) - (10) should be done between two adjacent zeros of the arm current, using the respective duty cycle function. The modulation function $m(t)$ in Table II is the modulation index that is proportional to the voltage being inserted by the arms:

$$
m(t)=\frac{v_{a r m}(t)}{N_{S M} \overline{V_{S M}}}=\frac{V_{D C_{a r m}}+v_{A C_{a r m}} \cos (\omega t)}{N_{S M} \overline{V_{S M}}}
$$

Table II: Duty cycles and current polarities on a HB-SM

\begin{tabular}{|c|c|c|}
\hline Device conducting & Current polarity & Duty cycle \\
\hline T1 & $i_{\text {arm }}<0$ & $\alpha(t)=m(t)$ \\
\hline D1 & $i_{\text {arm }}>0$ & $\alpha(t)=m(t)$ \\
\hline T2 & $i_{\text {arm }}>0$ & $\alpha(t)=1-m(t)$ \\
\hline D2 & $i_{\text {arm }}<0$ & $\alpha(t)=1-m(t)$ \\
\hline
\end{tabular}

If the SMs are FBs, a similar analysis can be done. The main difference, if no over modulation is considered, is that for bypassing the SM two actions are possible: to turn ON S1 and S3 or to turn ON S2 and S4 (Fig. 1b). If it is assumed that both actions happens evenly, i.e. $50 \%$ of the time each, the expressions (9) - (10) are still valid by using the duty cycles on Table III. Concerning the switching 
losses on each switch, they are given by the turn-on and turn-off energies. These energies can be estimated from a polynomial approximation as:

$$
\begin{gathered}
E_{O N_{I G B T}}=a_{o n}+b_{o n} i_{I G B T}+c_{o n} i_{I G B T}^{2} \\
E_{O F F_{I G B T}}=a_{o f f}+b_{o f f} i_{I G B T}+c_{o f f} i_{I G B T}^{2} \\
E_{\text {rec }} \text { Diode } \\
=a_{\text {rec }}+b_{\text {rec }} i_{\text {Diode }}+c_{\text {rec }} i_{\text {Diode }}^{2}
\end{gathered}
$$

Table III: Duty cycles and current polarities on a FB-SM

\begin{tabular}{|c|c|c|}
\hline Device conducting & Current polarity & Duty cycle \\
\hline T1 & $i_{\text {arm }}<0$ & $\alpha(t)=m(t)+0.5 \cdot(1-m(t))$ \\
\hline D1 & $i_{\text {arm }}>0$ & $\alpha(t)=m(t)+0.5 \cdot(1-m(t))$ \\
\hline T2 & $i_{\text {arm }}>0$ & $\alpha(t)=0.5 \cdot(1-m(t))$ \\
\hline D2 & $i_{\text {arm }}<0$ & $\alpha(t)=0.5 \cdot(1-m(t))$ \\
\hline T3 & $i_{\text {arm }}>0$ & $\alpha(t)=0.5 \cdot(1-m(t))$ \\
\hline D3 & $i_{\text {arm }}<0$ & $\alpha(t)=0.5 \cdot(1-m(t))$ \\
\hline T4 & $i_{\text {arm }}<0$ & $\alpha(t)=m(t)+0.5 \cdot(1-m(t))$ \\
\hline D4 & $i_{\text {arm }}>0$ & $\alpha(t)=m(t)+0.5 \cdot(1-m(t))$ \\
\hline
\end{tabular}

Where the coefficients can be determined from the device datasheet, and the currents are the current at the switching event. These currents are difficult to determine analytically due the stochastic behavior of the switching actions caused by the capacitor balancing algorithms [15]. However, assuming that with a given balancing algorithm the switching events are distributed uniformly over the fundamental AC cycle, the switching losses can be approximated [13], [15]. Under that hypothesis the switching losses are given by [13]:

$$
P_{\text {swithing }}=f_{\text {Sw avg }} \int_{0}^{T} E_{\text {ON IGBT }}\left(\left|i_{\text {arm }}(t)\right|\right)+E_{O F F I G B T}\left(\left|i_{\text {arm }}(t)\right|\right)+E_{\text {rec diode }}\left(\left|i_{\text {arm }}(t)\right|\right) d t
$$

This equation requires to know the average switching frequency of the SMs. Since the switching events are randomly allocated, it is difficult to obtain an analytical formula. Then in this study it is proposed to simulate each converter with a semi-analytical detailed model [16] using the "Average Tolerance Band technique" described in [17], to obtain the switching frequency for different cases. Finally, Eq. (15) is valid for both HB-SMs and FB-SMs [15].

\section{Front-to-Front MMC}

The arm expressions on an MMC in steady state are determined by $\mathrm{DC}$ and $\mathrm{AC}$ grid voltages and currents:

$$
\begin{gathered}
v_{u, l}=\frac{V_{D C}}{2} \mp v_{a c} \cos \omega t \\
i_{u, l}=\frac{I_{D C}}{3} \pm \frac{i_{a c}}{2} \cos (\omega t+\phi)
\end{gathered}
$$

In the F2F-MMC the DC quantities are the same, but the AC

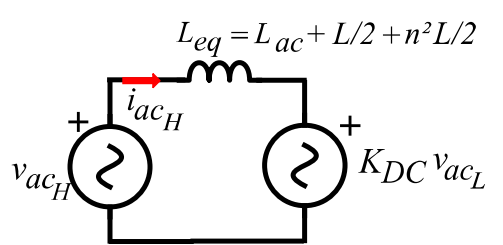

Fig. 2. F2F-MMC AC equivalent circuit.

quantities depend on the control method. Fig. 2 shows the converter AC equivalent circuit. To minimize the $\mathrm{AC}$ current it is proposed to fix the $\mathrm{AC}$ voltages to their maximum value and control the power transfer with the phase shift. The AC voltages can be expressed in function of the DC arm voltages:

$$
\widehat{v_{a c_{H}}}=k_{1} \frac{V_{H}}{2} \quad \widehat{v_{a c_{L}}}=k_{2} \frac{V_{L}}{2}
$$

A margin should be kept for dynamic purposes, so the value of the modulation indexes $k_{1}, k_{2}$ are selected to 0.85 in the present study. The AC power exchange between both MMCs is given by:

$$
P_{A C}=\frac{3}{2} \frac{\left(\widehat{v_{a c_{H}}} K_{D C} \widehat{v_{a c_{L}}} \sin \delta\right)}{2 \pi f L_{e q}}
$$


From this equation the phase angle between both $\mathrm{AC}$ voltages can be determined. The $\mathrm{AC}$ current is then calculated on the equivalent AC circuit of Fig. 2.

$$
i_{a c_{H}}=\frac{v_{a c_{H}} \angle \delta-K_{D C} v_{a c_{L}} \angle 0^{\circ}}{j 2 \pi f L_{e q}}=i_{a c_{H}} \angle \phi=-\frac{i_{a c_{L}}}{K_{D C}} \angle \phi
$$

So the F2F-MMC arm expressions are for each MMC on HV and LV sides:

$$
M M C_{H}\left\{\begin{array} { c } 
{ v _ { u , l } = \frac { V _ { H } } { 2 } ( 1 \mp k _ { 1 } \operatorname { c o s } ( \omega t + \delta ) ) } \\
{ i _ { u , l } = \frac { I _ { H } } { 3 } \pm i _ { a c _ { 1 } } \operatorname { c o s } ( \omega t + \phi ) }
\end{array} \quad M M C _ { L } \left\{\begin{array}{c}
v_{u, l}=\frac{V_{L}}{2}\left(1 \mp k_{2} \cos \omega t\right) \\
i_{u, l}=\frac{I_{L}}{3} \pm i_{a c_{2}} \cos (\omega t+\phi)
\end{array}\right.\right.
$$

The maximum voltage to be generated by each converter arm is, as in the MMC, equal to the DC grid voltage. Therefore, the number of submodules required per arm are:

$$
N_{S M a r m}{ }_{M M C_{\mathrm{H}}}=\frac{V_{H}}{\overline{V_{S M}}} \quad N_{S M a r m}{ }_{M M C_{L}}=\frac{V_{L}}{\overline{V_{S M}}}
$$

Fig. 3 shows the arm RMS current for both MMCs obtained directly from the expression of currents on Eq. (19) for the case study presented in Table I. This value is higher than the RMS on each SM switch (Eq. (10)), but is simpler to interpret. Fig. 3 also presents the SM capacitor sized according to Eq. (4) and the SM average switching frequency obtained from the detailed model simulation. These results are presented for different DC voltage transformation ratios and in function of the converter operation frequency.
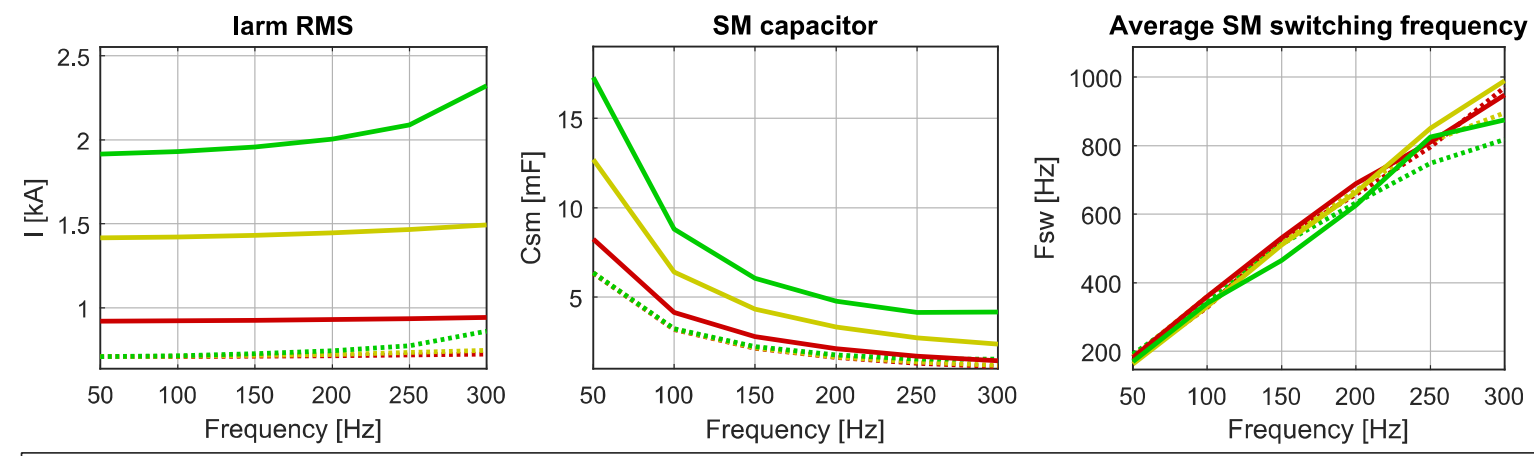

$\longrightarrow$ MMCL K $_{\mathrm{DC}}=1.3 \quad \ldots \ldots \ldots \mathrm{MMCH} \mathrm{K}_{\mathrm{DC}}=1.3$

MMCL K $\mathrm{DC}_{\mathrm{DC}}=2 \quad \ldots \mathrm{MMCH} \mathrm{K}_{\mathrm{DC}}=2$

MMCL K $_{\mathrm{DC}}=2.7 \quad \ldots \ldots . . \mathrm{MMCH} \mathrm{K}_{\mathrm{DC}}=2.7$

Fig. 3 - F2F-MMC: RMS arm currents, SM capacitor and Average SM switching frequency

According to these results, the arm currents increase slightly with the frequency. This is explained by the fact that the inductances on the circuit were kept constant for all the studied cases, then more reactive current flows in the circuit for high frequencies. Concerning the SM capacitor, it decreases in function of the frequency as expected from Eq. (4). The SM switching frequency shows a rather linear dependence with the operation frequency obtained from the detailed model circuit simulation.
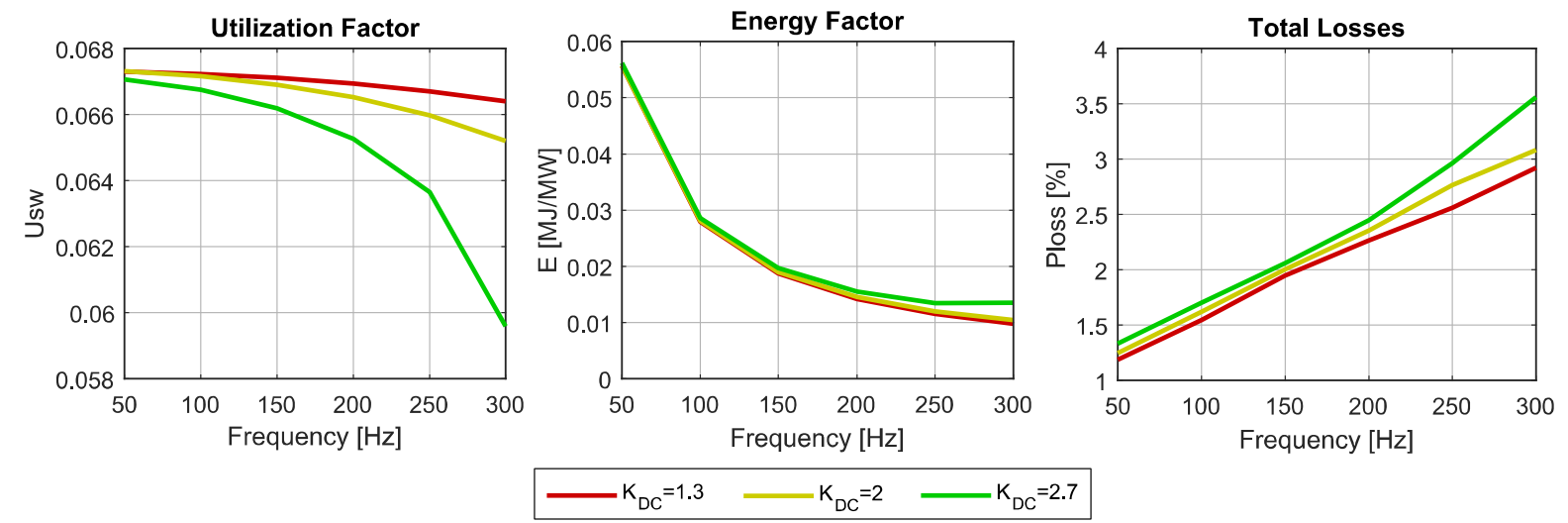

Fig. 4 - F2F-MMC performance indicators 
From these results it is expected that the utilization and factor decrease while the total losses increase with the frequency. These indicators are presented in Fig. 4 where these hypotheses are confirmed. Then for higher frequencies the F2F-MMC could experience a size reduction due to the reduction in the energy factor but at the expense of a slightly worst utilization of the semiconductors and with important increase of losses.

\section{DC-MMC}

From the analysis of one phase of the DC-MMC, the DC quantities of upper and lower arms are obtained:

$$
\begin{array}{cc}
v_{u_{D C}}=V_{H}-V_{L} & i_{u_{D C}}=\frac{I_{H}}{3} \\
v_{l_{D C}}=V_{L} & i_{l_{D C}}=\frac{I_{H}-I_{L}}{3}
\end{array}
$$

Concerning the $\mathrm{AC}$ voltages, it is assumed that upper and lower arm generate the same $\mathrm{AC}$ voltages but in phase opposition. The value depends on the DC arm voltages. Since no over-modulation is considered, the $\mathrm{AC}$ voltages depend only on the $\mathrm{DC}$ conversion ratio:

$$
\widehat{v_{a c}}=\left\{\begin{array}{cc}
k\left(V_{H}-V_{L}\right) & K_{D C}<2 \\
k V_{L} & K_{D C}>2
\end{array}\right.
$$

Where $k$ is fixed to 0.85 , leaving a margin for dynamic purposes as in the case of the F2F-MMC. To calculate the AC currents, the needed AC circulating power is first estimated. The AC power is needed to balance the energy on the converter arms. Analyzing the arm DC powers, it is observed that the upper arm is being charged/discharged at the same ratio that the lower arm:

$$
P_{u_{D C}}=\frac{\left(V_{H}-V_{L}\right) I_{H}}{3}=\frac{P_{D C}}{3}\left(1-\frac{1}{K_{D C}}\right) \quad P_{l_{D C}}=\frac{V_{L}\left(I_{H}-I_{L}\right)}{3}=\frac{P_{D C}}{3}\left(\frac{1}{K_{D C}}-1\right)
$$

Therefore, to achieve the energy balanced the AC power to be circulated in one converter leg is:

$$
P_{A C}=\frac{P_{D C}}{3}\left(1-\frac{1}{K_{D C}}\right)
$$

The equivalent $\mathrm{AC}$ circuit of the DC-MMC is presented in Fig. 5. Doing circuit analysis, the expression of the power exchange between arms is obtained:

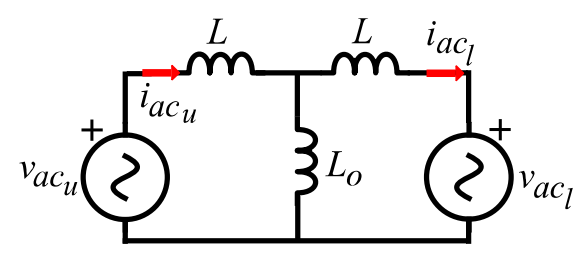

Fig. 5 - DC-MMC AC Equivalent Circuit

$$
P_{A C}=\frac{L_{o}}{L\left(L+2 L_{o}\right)} \frac{\widehat{v_{u}} \widehat{v}_{l} \sin \delta}{2 \cdot 2 \pi f}
$$

With this expression the phase angle between arms can be calculated and the AC current is determined.

$$
\left(\begin{array}{c}
i_{u_{a c}} \angle \phi_{u} \\
i_{l_{a c}} \angle \phi_{l}
\end{array}\right)=-\frac{1}{j 2 \pi f L\left(L+L_{o}\right)}\left(\begin{array}{cc}
L+L_{o} & L_{o} \\
L_{o} & L+L_{o}
\end{array}\right)\left(\begin{array}{c}
v_{u_{a c}} \angle \delta \\
v_{l_{a c}} \angle 0
\end{array}\right)
$$

So the DC-MMC arms expressions in steady-state are:

$$
\begin{array}{cc}
v_{u}=V_{H}-V_{L}-\widehat{v_{a c}} \cos (\omega t+\delta) & i_{u}=\frac{I_{H}}{3}+i_{u a c} \cos \left(\omega t+\phi_{u}\right) \\
v_{l}=V_{L}+\widehat{v_{a c}} \cos (\omega t) & i_{l}=\frac{I_{H}-I_{L}}{3}+i_{l_{a c}} \cos \left(\omega t+\phi_{l}\right)
\end{array}
$$

The maximum voltage to be generated by each arm is $V_{D C_{a r m}}+\widehat{v_{a c}}$. However under faults the voltage to be withstood is different. In case of a fault on low voltage side, the upper arms have to withstand $V_{H}$. Additionally, if the fault is on HV side, the upper arms need FB-SMs to block the fault. The quantity of FBs must be enough to generate a voltage of $-V_{L}$. Then, the required number of SMs on each arm is:

$$
N_{S M s_{l}}=\left\{\begin{array}{ccc}
\frac{V_{H}}{\overline{V_{S M}}} & K_{D C}<2 \\
\frac{V_{L}}{\overline{V_{S M}}} & K_{D C}>2
\end{array} \quad N_{S M s u}=\left\{\begin{array}{cc}
\frac{V_{L}}{\overline{V_{S M}}} \mathrm{FBs}+\frac{V_{H}-V_{L}}{\overline{V_{S M}}} \text { HBs } & \text { if blocking capability } \\
\frac{V_{H}}{\overline{V_{S M}}} \mathrm{HBs} & \text { if no blocking capability }
\end{array}\right.\right.
$$


The RMS arm currents, SM capacitors and switching average frequency are calculated for different cases and presented in Fig. 6. As in the case of the F2F-MMC the presented currents are the arm values and not on the individual switches. It should be noted that although the DC-MMC upper arm has two kind of SMs the sized capacitor is the same for the FBs and HBs on the arm, then only one value for the upper arm capacitor is showed.
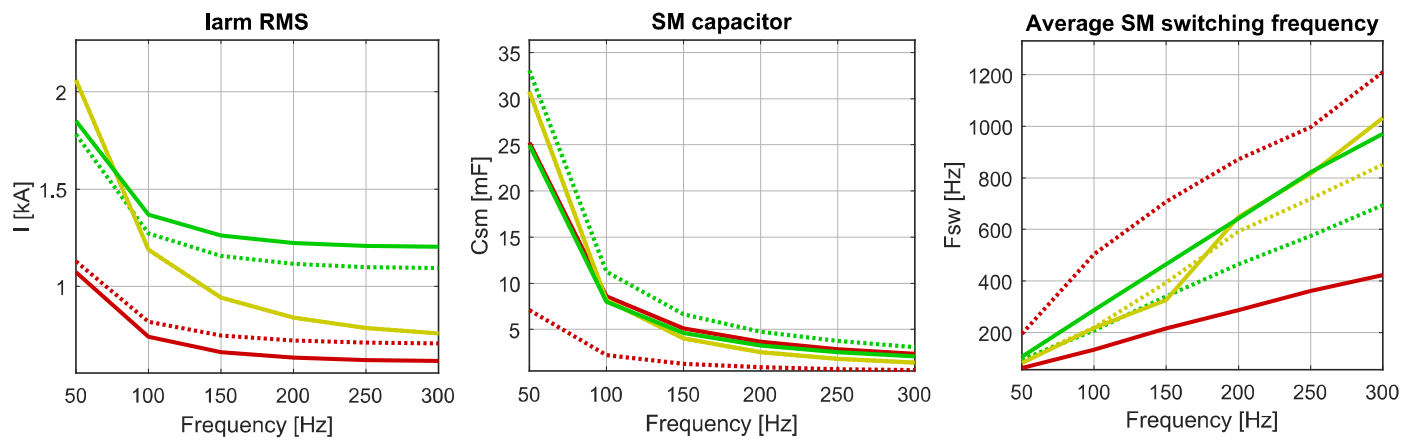

\begin{tabular}{|c|c|c|}
\hline Lower Arm $\mathrm{K}_{\mathrm{DC}}=1.3$ & Lower Arm $\mathrm{K}_{\mathrm{DC}}=2$ & Lower Arm K $\mathrm{DC}=2.7$ \\
\hline$\ldots \ldots \ldots$ Upper Arm $\mathrm{K}_{\mathrm{DC}}=1.3$ & . & $\ldots \ldots$ Upper Arm $K_{D C}=2.7$ \\
\hline
\end{tabular}

Fig. 6 - DC-MMC: RMS arm current, SM capacitor and Average SM switching frequency

As seen, in the DC-MMC the arm currents decrease with the increase of the frequency. The reason is that at higher frequencies the output filter inductors present a higher impedance decreasing the value of the AC current. The SM capacitors also decrease as expected by Eq. (4). According to the detailed model simulation the switching frequency increases with the operation frequency.

Fig. 7 shows the DC-MMC performance indicators. It is seen that at higher frequencies the semiconductor utilization increases and the energy factor decreases. The reason comes from the results in Fig. 6: at higher frequencies the RMS currents and the SM capacitances decrease. Concerning the losses, it is seen that they decrease until some frequency value and then start to increase. The reason of this behavior is from the separation of conduction and switching losses, presented on Fig. 8 where the decrease on the conduction losses is given mainly by the reduction on the arm currents, while the increase on switching losses is given by the increase of switching frequency.
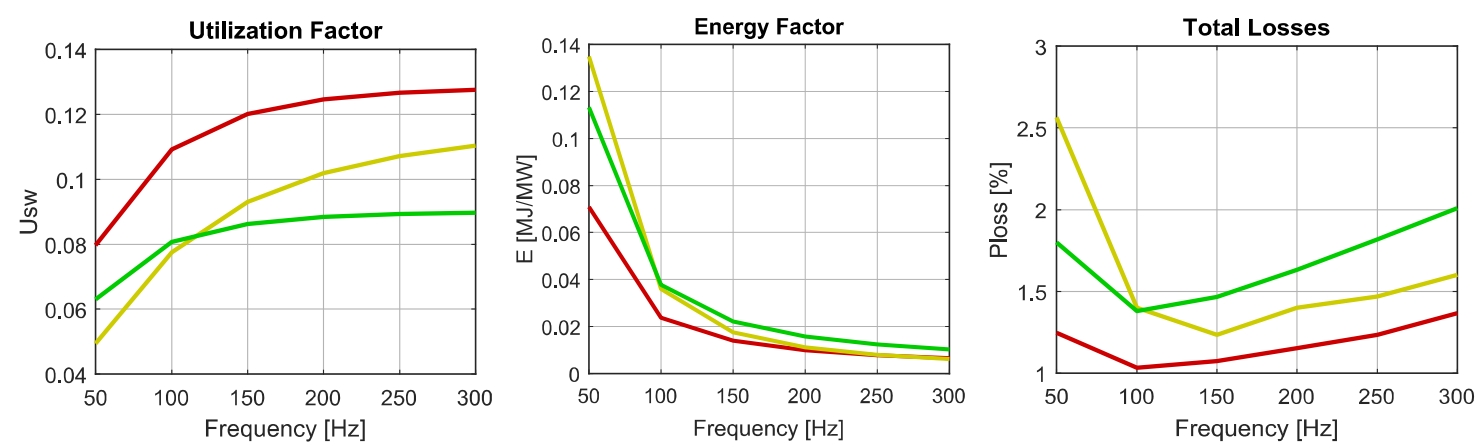

Fig. 7 - DC-MMC performance indicators
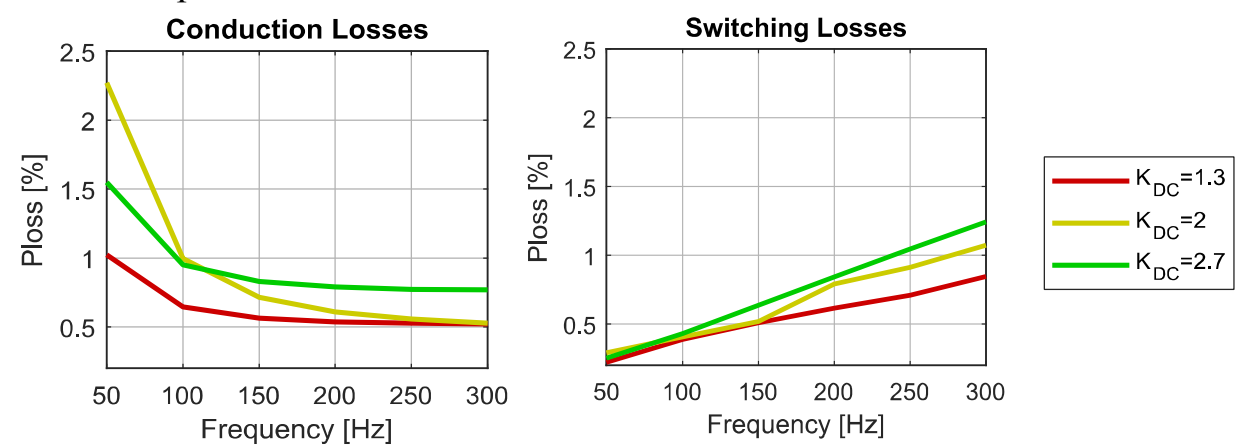

Fig. 8 - DC-MMC conduction and switching losses 


\section{Converter comparison}

It is proposed to compare the circuits using the selected key performance indicators since they can be used to make a quick assessment of the potential benefits of each circuit for each particular case. The indicators can be grouped into two graphs: an Energy vs Utilization factor chart, and a Switching vs Conduction losses chart. These graphs are presented in Fig. 9. The "best converter" candidates in regard of the analyzed indicators would be placed in the bottom-right corner for the first chart, i.e. less energy installed and higher utilization of semiconductors, so potentially less converter size and less investment in semiconductors. For the losses chart the "best circuit" would be closer to the origin.

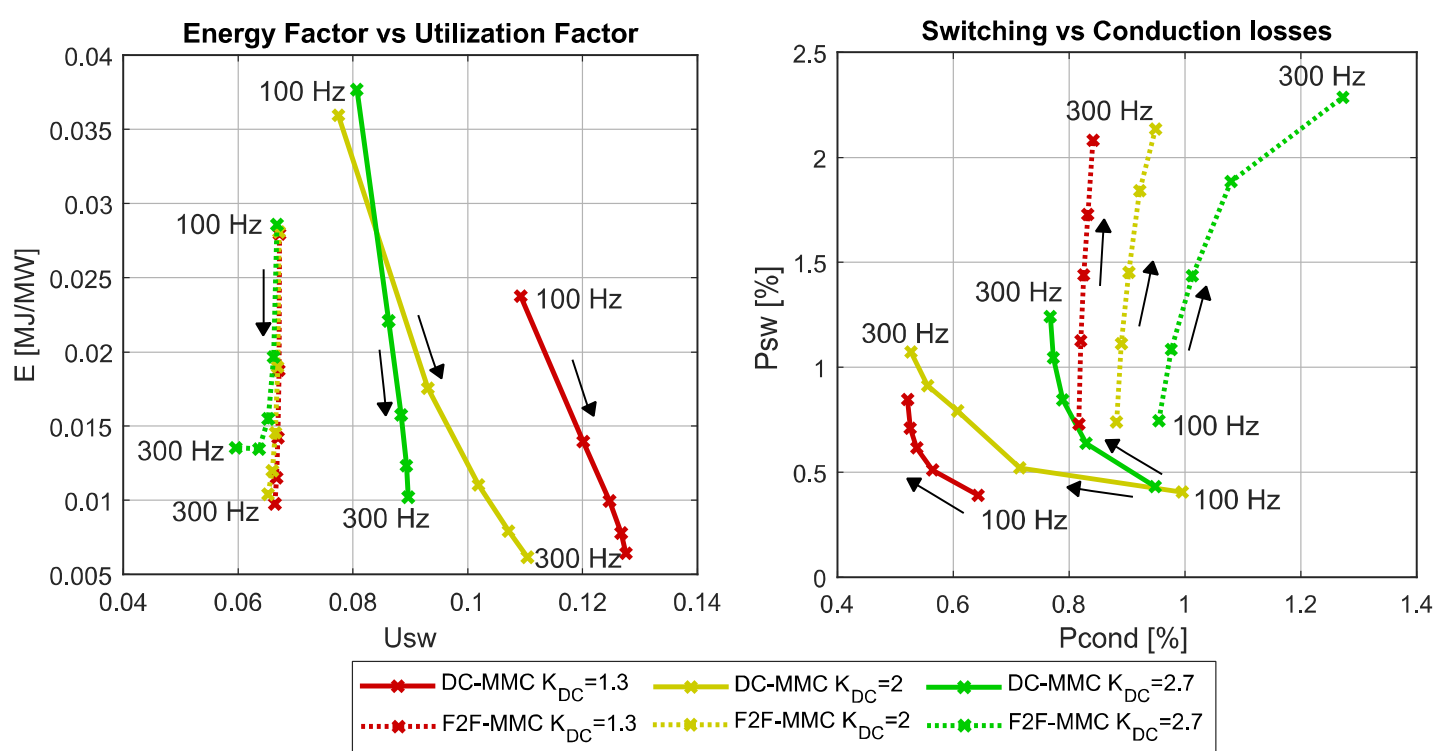

Fig. 9 - Comparison of F2F-MMC and DC-MMC. (The X marks represent the operation frequencies in steps of $50 \mathrm{~Hz}$ and the arrows point in direction of the increase of operation frequency)

From these charts it becomes clear that the DC-MMC presents a better performance for all the cases compared to the F2F-MMC. The converter installed energy decreases for both converters when the operation frequency increases while the utilization of semiconductors does not change significantly for the F2F-MMC but for the DC-MMC it does. Concerning the converter losses, the F2F-MMC is strongly affected by the increase of the frequency mainly due to the switching losses. The DC-MMC is less affected on its switching losses while the conduction losses are reduced. For the case of $K_{D C}=2$ the operation frequency has a greater impact on the DC-MMC indicators compared to the other cases.

It seems that there is an optimum frequency for the $\mathrm{DC}-\mathrm{MMC}$ when the losses are minimal, the increase on the utilization factor and the reduction on the energy factor are significant. To find this value a more detailed study should be done to establish the relationship between the proposed indicators and the converter cost.

\section{Conclusion}

This paper proposes a general methodology with three main indicators to compare modular multilevel HVDC DC-DC converters. It has been applied to study the impact of the operating apparent internal frequency on two structures, but it can be used to study the sensibility to other parameters. The increase in the frequency showed a decrease in the converter installed energy and so potentially the converter size, at the expense of increasing the switching losses. The utilization of semiconductors and the conduction losses relation with the internal frequency depend strongly on the structure, for the F2FMMC these indicators are degraded while for the DC-MMC they are improved. The optimal choice of the internal operation frequency depends on how the assessment factors affect the real cost and size of the converter. However as seen, the proposed methodology can be used to rapidly estimate the interest of one topology compared to another one. The validity of all analytical expressions used in the paper were validated in simulation with high degree of reliability, except for the switching losses that showed some differences depending on the implemented BCA parameters. Some key indicators should be 
equally proposed in future works to evaluate the passive elements in the circuit, such as AC transformers, passive filters and reactors.

\section{References}

[1] D. Jovcic, D. Van Hertem, K. Linden, J.-P. Taisne, and W. Grieshaber, "Feasibility of DC transmission networks," in Innovative Smart Grid Technologies (ISGT Europe), 2011 2nd IEEE PES International Conference and Exhibition on, 2011, pp. 1-8.

[2] J. D. Páez, J. Maneiro, D. Frey, S. Bacha, A. Bertinato, and P. Dworakowski, "Study of the impact of DC-DC converters on the protection strategy of HVDC grids," in 15th IET International Conference on AC and DC Power Transmission (ACDC 2019), 2019, pp. 1-6.

[3] J. D. Páez, D. Frey, J. Maneiro, S. Bacha, and P. Dworakowski, "Overview of DC-DC converters dedicated to HVdc grids," IEEE Transactions on Power Delivery, vol. 34, no. 1, pp. 119-128, 2019.

[4] W. Chen, A. Q. Huang, C. Li, G. Wang, and W. Gu, "Analysis and comparison of medium voltage high power DC/DC converters for offshore wind energy systems," IEEE Transactions on Power Electronics, vol. 28, no. 4, pp. 2014-2023, 2013.

[5] A. Schön and M.-M. Bakran, "Comparison of modular multilevel converter based HV DC-DCconverters," in Power Electronics and Applications (EPE'16 ECCE Europe), 2016 18th European Conference on, 2016, pp. 1-10.

[6] A. Schön and M.-M. Bakran, "Comparison of the most efficient DC-DC converters for power conversion in HVDC grids," in PCIM Europe 2015; International Exhibition and Conference for Power Electronics, Intelligent Motion, Renewable Energy and Energy Management; Proceedings of, 2015, pp. 1-9.

[7] S. Kenzelmann, A. Rufer, D. Dujic, F. Canales, and Y. R. De Novaes, "Isolated DC/DC structure based on modular multilevel converter," IEEE Transactions on Power Electronics, vol. 30, no. 1, pp. 89-98, 2015.

[8] J. A. Ferreira, "The multilevel modular DC converter," IEEE Transactions on Power Electronics, vol. 28 , no. 10 , pp. 4460-4465, 2013.

[9] S. Norrga, L. Ängquist, and A. Antonopoulos, "The polyphase cascaded-cell DC/DC converter," in Energy Conversion Congress and Exposition (ECCE), 2013 IEEE, 2013, pp. 4082-4088.

[10] G. J. Kish, "On the Emerging Class of Non-Isolated Modular Multilevel DC-DC Converters for DC and Hybrid AC-DC Systems," IEEE Transactions on Smart Grid, vol. 10, no. 2, pp. 1762 1771, Mar. 2019.

[11] R. Marquardt, A. Lesnicar, and J. Hildinger, "Modulares stromrichterkonzept für netzkupplungsanwendung bei hohen spannungen," ETG-Fachtagung, Bad Nauheim, Germany, vol. 114, 2002.

[12] L. M. Cunico, G. Lambert, R. P. Dacol, S. V. G. Oliveira, and Y. R. de Novaes, "Parameters design for modular multilevel converter (MMC)," in Power Electronics Conference (COBEP), 2013 Brazilian, 2013, pp. 264-270.

[13] P. Ladoux, P. Marino, G. Raimondo, and N. Serbia, "Comparison of high voltage modular AC/DC converters," in International Symposium on Power Electronics Power Electronics, Electrical Drives, Automation and Motion, 2012, pp. 843-848.

[14] Z. Zhang, Z. Xu, and Y. Xue, "Valve losses evaluation based on piecewise analytical method for MMC-HVDC links," IEEE Transactions on Power Delivery, vol. 29, no. 3, pp. 1354-1362, 2014.

[15] P. S. Jones and C. C. Davidson, "Calculation of power losses for MMC-based VSC HVDC stations," in 2013 15th European Conference on Power Electronics and Applications (EPE), 2013, pp. 1-10.

[16] A. Zama, S. Bacha, A. Benchaib, D. Frey, and S. Silvant, "A novel modular multilevel converter modelling technique based on semi-analytical models for HVDC application," J. Elect. Syst., vol. 12, no. 4, pp. 649-659, 2016.

[17] A. Zama, S. A. Mansour, D. Frey, A. Benchaib, S. Bacha, and B. Luscan, "A comparative assessment of different balancing control algorithms for modular multilevel converter (MMC)," in 2016 18th European Conference on Power Electronics and Applications (EPE'16 ECCE Europe), 2016, pp. 1-10. 